\title{
Problemas sobre la contrastación de teorías en su representación modelística*
}

Problems on Theory Testing in their Model Representation

Juan Manuel Jaramillo Uribe $e^{\dagger}$

\begin{abstract}
Resumen
Con la presentación/identificación semántica o modelo-teórica de las teorías empíricas, como alternativa a la presentación/identificación enunciativa y/o lingüística, establecer el valor veritativo de ellas se torna problemático, pues la verdad y la falsedad son propiedades de los enunciados y no de las estructuras y/o modelos. El enunciado Ramsey-Sneed constituye para los que sostienen el estructuralismo metateórico una adecuada solución a tal problema. A fin de clarificar la propuesta modelo-teórica estructuralista, se la comparará con la realizada por Bas van Fraassen dentro del enfoque de espacio de estados.

Palabras clave: contrastación empírica - concepción modelística - enfoque de espacio de estados - estructuralismo metateórico - enunciado Ramsey-Sneed
\end{abstract}

\begin{abstract}
With the semantic or model-theoretical presentation/identification of empirical theories, as an alternative to the statement and/or linguistic presentation/identification, establishing the truth value of them becomes problematic, considering truth and falsehood are properties of statements and not of structures or models. The Ramsey-Sneed statement constitutes for supporters of metatheoretical structuralism an adequate solution to such problem. In order to clarify the structuralist model-theoretical proposal, it will be compared with the one made by Bas van Fraassen within the state-space approach.

Keywords: empirical testing - modelistic view - state-space approach - metatheoretical structuralism - Ramsey-Sneed statement
\end{abstract}

\footnotetext{
* Recibido: 11 de noviembre de 2012. Aceptado con revisiones: 5 de marzo de 2013.

+ Universidad del Valle, Colombia. Para contactar al autor, por favor, escribir a: jaramillo.juanmanuel@gmail.com. Metatheoria 5(2)(2015): 85-97. ISSN 1853-2322. eISSN 1853-2330.

(C) Editorial de la Universidad Nacional de Tres de Febrero. Publicado en la República Argentina.
} 


\section{Introducción}

El principio de propagación de cuestiones propuesto por I. Kant según el cual la solución o disolución de cualquier problema engendra otros problemas adquiere su plena vigencia no sólo en la ciencia, sino también en la filosofía y, como veremos, en la filosofía de la ciencia que, como disciplina institucional, apenas tiene poco más de un siglo de existencia. En estos casos, las propuestas de solución a problemas legítimos se vuelven imperativas y no es menester acudir a la estrategia de los insolubilia o de los problemas insolubles.

En este escrito me propongo mostrar que si bien la presentación/identificación de las teorías empíricas por parte de la concepción semántica o modelística (como preferiré llamarla) como familias, clases o conjuntos de modelos constituye una verdadera alternativa a la propuesta metodológica exclusivamente sintáctico-formal de la filosofía clásica de la ciencia, también hay decir que con ella se originan una serie de problemas metateóricos no menos importantes como son, entre otros, los que tienen que ver con las relaciones entre la representación de los modelos y lo representado por ellos que focalizaron la atención de los filósofos de la ciencia en las dos últimas décadas del siglo XX y que aún siguen siendo objeto de discusión y de análisis. Tal asunto lleva aparejada una serie de cuestiones de carácter ontológico, epistemológico, metodológico y semántico, amén de todas aquellas de carácter pragmático que, como veremos, tienen que ver con el uso de los modelos (modelos teóricos) en situaciones experimentales y de laboratorio.

No obstante, el análisis de estas cuestiones dependerá, en últimas, del tipo de perspectiva metateórica que se adopte. Un realista, por ejemplo, aceptaría que los modelos, al no ser entidades lingüísticas, no son per se verdaderos o falsos, pero las descripciones/explicaciones que con ellos hagamos, así sean de fenómenos idealizados, sí lo son y esto implica la creencia de que la teoría científica es verdadera (realismo alético). Otro tanto podría decirse sobre los relata de los términos singulares (individuos) y de los predicados (propiedades) que aparecen en el lenguaje de las teorías científicas con cuya existencia, independientemente de las teorías y como algo prefabricado, un realista, como el realista científico, se compromete ontológicamente, pero que para un antirrealista como van Fraassen resultarían problemáticos en la medida en que su compromiso (ontológico) se circunscribe únicamente a los fenómenos observables, dentro claro, está, de un antropocéntrico y problemático noción de lo que, para él, es 'observable'. En el caso de la ciencia esto reviste para él particular importancia, pues el contenido de la representación del mundo que ella nos provee es mucho más rico que lo que el ojo humano discierne o puede discernir. Ella misma -apostilla van Fraassen- distingue lo observable que postula, del todo que postula. En otras palabras, mientras el 'realista' asume un compromiso ontológico respecto de los referentes de todos los términos singulares y de todos los predicados de una teoría, el antirrealista no se compromete con dicha existencia o, al menos -como en el caso del van Fraassen- con la existencia de los referentes no observables de dichos términos. Para van Fraassen la aceptación de una teoría sólo implica la creencia de que ella es empíricamente adecuada, en el sentido de creer que 'salva los fenómenos', i.e., que "describe correctamente lo que es observable", de suerte para presentar/identificar modelísticamente una teoría, este autor considere la necesidad de

especificar una familia de estructuras, sus modelos, y, en segundo lugar, [...] ciertas partes de esos modelos (las subestructuras empiricas) como candidatos para la representación directa de los fenómenos observables (van Fraassen 1996, p. 89).

De todo esto se sigue que aunque la posición de este autor tipifica una forma de 'antirrealismo' que Moulines (2006, p. 125) considera como una variante del instrumentalismo, hay que advertir, sin embargo, que no se trata de un 'instrumentalismo puro', sino de un 'instrumentalismo débil', pues el autor admite un sentido en el que se puede suponer que los modelos de una teoría son literalmente verdaderos con relación al mundo real, i.e., cuando la teoría, como él dice, 'salva los fenómenos"”. 
Modelísticamente hablando, este caso se presenta cuando existe un modelo teórico tal que todos los fenómenos observables 'encajan' o, mejor, son 'isomorfos' -como lo expresa van Fraassen- a subestructuras de ese modelo. En este caso estamos hablando de subestructuras de estructuras que constituyen los modelos; subestructuras que -como diría Quine- están subdeterminadas o infradeterminadas por las observaciones.

Sin embargo, más allá de la cuestión de la adecuación empírica de las teorías que concierne a la relación entre la teoría y el mundo, existen otras cuestiones relativas al uso y a la utilidad de las teorías de índole pragmática y donde valores como la elegancia, la sencillez, el alcance, la completitud, etc. de las teorías son valores que ofrecen razones para usar o preferir una teoría, "independientemente de las preguntas por la verdad" (Moulines 2006, p. 117).

Aunque a partir de la década de lo 80 buena parte de los estudios en filosofía de la ciencia se focalizan en las relaciones de las teorías científicas con el mundo (aspecto semántico) y en el papel de los usuarios de las teorías (aspecto pragmático), sin embargo, hay que reconocer que la preocupación por los problemas de la estructura y del contenido de las teorías científicas no fue menos importante, como se evidencia, por ejemplo, en el enfoque modelístico de presentación/identificación de las teorías.

A continuación me ocuparé de este problema en dos de los casos que, en mi opinión, son los más representativos de la concepción modelística de las teorías científicas desarrollada durante las tres últimas décadas del siglo XX y de lo que va del siglo XXI: el empirismo constructivo de Bas C. van Fraassen y el estructuralismo metateórico iniciado por Joseph D. Sneed con la publicación de su obra The Logical Structure of Mathematical Physics (1971).

\section{2.}

Pese a que todos los representantes de la concepción modelística o semántica de las teorías comparten la estrategia (metodológica) de presentar/identificar las teorías como una clase o conjunto de modelos, ${ }^{1}$ sin embargo, entre ellos no existe un consenso en torno a la naturaleza matemática de dichos modelos. Van Fraassen y F. Suppe (siguiendo a H. Weyl y a E. Beth), por ejemplo, identifican los modelos con 'puntos' o 'trayectorias' en un espacio de estados o de fases, como, en general, se hace en los manuales de física $^{2}$ donde los estados de los sistemas físicos se identifican con puntos o trayectorias en un determinado sistema de coordenadas y donde el número de dimensiones del espacio de estados es idéntico al número de componentes (parámetros o funciones) ${ }^{3}$ que determinan, en cada caso, el estado del sistema. Así, a cada tipo de sistema físico corresponde un espacio de estados como el conjunto de todas las posibles n-secuencias de valores, donde $n$ es la dimensión del espacio. Claro está que en el formalismo matemático de la mecánica cuántica, el espacio de estados o de fases clásico se reemplaza por un espacio abstracto distinto conocido como espacio de Hilbert donde los estados puros de un sistema físico matemáticamente se representan en el espacio de Hilbert por funciones de onda o por vectores de longitud unaria donde la función de onda asocia a cada propiedad de un sistema físico un valor de probabilidad, obteniéndose así un situación semántica polivalente en la que la validez del clásico principio del tercer excluido se torna problemática. En este caso, al menos, caben tres posibilidades:

1) Un estado puro $s$ verifica una propiedad $P$, porque asocia a $P$ un valor de probabilidad 1 ;

2) el estado puro $s$ falsa $P$, porque $s$ asocia a $P$ un valor de probabilidad 0 ;

\footnotetext{
${ }^{1}$ Aquí, no obstante, vale la pena recordar una aclaración hecha por van Fraassen en el sentido que si bien "una teoría científica no es identificable con ("with") una clase de modelos, sí es, sin embargo, identificable a través ("through") de ella, concebida propiamente" (van Fraassen 1985, p. 25).

${ }^{2}$ El método de espacios de estado es más próximo a la forma como las teorías físicas se presentan en los manuales de física, al menos en los de mecánica clásica y cuántica, pues es menos evidente en otras teorías donde los conceptos fundamentales no son magnitudes como en teorías químicas, biológicas y de ciencias sociales o, incluso, en teorías físicas como la teoría de la relatividad, como lo reconoció el mismo van Fraassen.

3 A estos parámetros o funciones los físicos, en general, suelen llamarlos "observables" en el sentido de que son propiedades de los sistemas físicos para las que existen procedimientos experimentales de medición, cuyos resultados se expresan en valores numéricos.
} 
3) en el estado puro $s$ la propiedad $P$ permanece indeterminada, porque $s$ asocia a $P$ un valor de probabilidad distinto tanto de 1 como de 0 .

Tertium datur! (se da un tercer caso) (Dalla Chiara \& Toraldo di Francia 2001, p.141).

Dentro del enfoque de espacio de estados, la presentación/identificación de las teorías concretas no se hace apelando a una formulación canónica que todas las teorías debe satisfacer, si bien, de modo general, se apela a un espacio $n$-dimensional que, en cada caso, se configura al introducir los axiomas propios (postulados o leyes) de la teoría. La única exigencia es que, en el espacio de estados, se representen, de modo abstracto, los estados posibles de los sistemas físicos o, para ser más precisos, las abstracciones e idealizaciones de ellos, pues aunque el campo de aplicación de las teorías empíricas es un dominio de fenómenos empíricos o una clase de éstos, su determinación se hace con base en un conjunto de parámetros seleccionados por la teoría, de suerte que lo que tenemos, como contraparte de la teoría, no son sistemas físicos en los que realmente intervienen muchos parámetros, sino una representación abstracta, idealizada y contrafáctica de ellos como si en ellos sólo intervinieran los parámetros seleccionados y como si se tratara de sistemas aislados, como sucede en las investigaciones experimentales y de laboratorio. Dentro de este enfoque (aplicable sólo a sistemas dinámicos y a teorías cuantitativas) la función de las diferentes leyes (como leyes de los modelos y no de la naturaleza) es imponer constricciones sobre las relaciones entre estados lógicamente posibles, a fin de poder establecer cuáles de éstos son nómicamente posibles: las leyes de coexistencia seleccionan, dentro de los estados lógicamente posibles de un sistema, el subconjunto de estados física o realmente posibles (la ley de Boyle establece como constricción nómica que de todos los tríos de valores que representan los valores de 'presión', 'volumen' y 'temperatura', respectivamente, mediante los cuales determinamos el estado de un sistema termodinámico, sólo son física o realmente posibles aquellos en los que $p \cdot v / t=R$, donde $R$ es un constante que depende de la cantidad y naturaleza del gas; las leyes de sucesión establecen, las trayectorias fisica o realmente posibles en un espacio de estados (historia del sistema), ${ }^{4} \mathrm{y}$, finalmente, las leyes de interacción fijan las interrelaciones recíprocas real o físicamente posibles entre los sistemas como un único sistema complejo.

Refiriéndose a la mecánica cuántica, van Fraassen expresa:

En el caso de la mecánica cuántica, los estados del sistema [como posibles estados] son representados por elementos (vectores de estado) de un espacio de Hilbert. Para cada magnitud física mensurable ("observable") $m$ existe un operador lineal $\mathrm{M}$ sobre este espacio, tal que $m$ tiene valor $r$ si y sólo si $\mathrm{Mx}=$ $r x$, donde $x$ es el vector estado ("statevector") que representa el estado del sistema (van Fraassen 1979, p. 329).

Y en una nota aclara:

Si $M x=r x$, llamaremos $r$ un valor propio ("eigenvalue") de $\mathrm{M}$ y a $x$ el vector propio correspondiente (van Fraassen 1979, p. 329). ${ }^{5}$

De conformidad con esto, para van Fraassen los modelos se identifican con el par ordenado $M=\langle X$, loc $\rangle$ donde ' $X$ ' es un conjunto de sistemas físicos idealizados y 'loc' una función de 'localización' que le asigna, a cada sistema o conjunto de sistemas físicos idealizados, una posición en el espacio de estados $H$; posición que, como vimos, depende de los valores de los parámetros definientes en un momento determinado. Pero tal localización (en el espacio de estados) no constituye un modelo teórico en sentido estricto, pues no indica su evolución o trayectoria; los modelos teóricos (a través de las leyes) tienen como función representar las trayectorias físicamente posibles de los sistemas físicos idealizados en

\footnotetext{
${ }^{4}$ Aquí conviene aclarar que, dependiendo del modo como describamos las leyes, las de coexistencia pueden verse como de sucesión y viceversa. Así, la ley de Boyle determina que un estado $\langle p, v, t\rangle$ (que siempre es estado de un sistema) puede transitar a otro posterior $\left\langle p^{\prime}\right.$, $\left.v^{\prime}, t^{\prime}\right\rangle$ syss la cantidad $p \cdot v / t$ es la misma en ambos. Lo mismo sucede con las leyes de sucesión que pueden ser vistas como de coexistencia si en éstas se introduce el parámetro temporal como constituyente de los estados del sistema.

${ }^{5}$ En la mecánica cuántica el estado cuántico es un concepto puramente matemático y abstracto y el estado cuántico no es el estado en el que se puede encontrar un objeto cuántico, pues al observar un objeto cuántico se establece siempre un valor propio ("eigenvalue") para ese observable, aunque el estado del sistema no sea un estado propio para ese observable.
} 
espacios de estados. En el caso de un gas, por ejemplo, el triplo de números reales de 'presión', 'temperatura' y 'volumen' representan los posibles estados termodinámicos del gas, pero la ley de Boyle establece un posible estado físico del gas syss ' $p \cdot v=R \cdot t$ ', donde $R$ es una constante que depende de la cantidad y naturaleza del gas. Las trayectorias físicas o realmente posibles (como una subclase de las trayectorias lógicamente posibles) es la secuencia de estados que puede asumir un sistema físico, determinada por las leyes de la teoría, en la medida en que son ellas las que establecen cómo se comportarían los fenómenos (aspecto contrafáctico) caso de que se dieran las condiciones ideales. Gracias a ellas, las teorías satisfacen las exigencias teóricas de explicación y de predicción. Siendo así, "cada modelo teórico consta de un dominio formado por la clase de los estados lógicamente posibles [mal llamado 'ontología' de la teoría] ${ }^{6}$ y una serie de atributos, que son leyes, los cuales indican qué estados son físicamente posibles a la luz de la teoría” (Perdomo \& Sánchez 2003, p. 127).

El reconocimiento de que de todos los estados, trayectorias y comportamientos de interacción lógicamente posibles, sólo algunos de ellos son físicamente posibles, conduce a van Fraassen a introducir, dentro de su teoría (semántica) de los lenguajes-semi-interpretados la función de "satisfacción" que conecta los modelos matemáticos (van Fraassen los llama "modelos teóricos) con los resultados de medición empíricos expresados mediante elementales (atómicos) $U$ de una teoría, de suerte que "Un enunciado elemental $U$ es verdadero respecto de $M=\langle X$, loc $\rangle$ syss $\operatorname{Loc}(X)=h(U)$ " (van Fraassen 1995a, p. 335). En este contexto, un lenguaje-semi-interpretado de una teoría es un triplo ordenado $L=\langle E, H, h\rangle$.

Por su parte, el programa estructuralista de reconstrucción de teorías propone, en contraste con el enfoque de espacio de estados, hace una caracterización de los modelos en términos de estructuras conjuntistas (o mejor, de "especie de estructuras conjuntistas, en el sentido de Bourbaki), ${ }^{7}$ lo que permite hablar de enfoque conjuntista o teórico-conjuntista. Dentro de este enfoque, teorías complejas como la mecánica newtoniana, la termodinámica de sistemas simples, la genética mendeliana o la teoría neo-clásica general del equilibrio (por mencionar sólo algunos casos representativos), se consideran como redes teóricas (normalmente cada red teórica se representan por $\mathrm{N}$ ) en las que se conectan diferentes elementos teóricos parcialmente ordenados en relaciones de especialización. Así, $\mathbf{N}$ $=\left\langle\left\{\mathbf{T}_{i}\right\}, \sigma\right\rangle$ es una red teórica syss def $(1)\left\{\mathbf{T}_{i}\right\}$ es un conjunto no vacío de elementos teóricos, y (2) $\sigma$ es una relación de especialización sobre $\left\{\mathrm{T}_{i}\right\}$. En términos generales se puede decir que esta noción de 'red teórica' es la que mejor expresa la naturaleza sincrónica o estática de las teorías científicas y corresponde a la imagen que de la ciencia nos proporcionan Kuhn y Lakatos, cuando se dejan de lado sus aspectos pragmáticos.

Cada elemento teórico $\mathrm{T}$ de la red está caracterizado por su núcleo $\mathrm{K}$ y un rango de aplicaciones intencionales, $\mathbf{I}$, tal que $\mathbf{T}=\langle\mathbf{K}, \mathbf{I}\rangle$. El núcleo $\mathbf{K}$ consta básicamente de los siguientes componentes como constituyentes esenciales de cualquier teoría empírica: $\mathbf{M}_{\mathrm{p}}$ (el conjunto de modelos potenciales), $\mathbf{M}$ (el conjunto de modelos), $\mathbf{M}_{\mathrm{pp}}$ (el conjunto de modelos parciales), $\mathbf{C}$ (el conjunto de condiciones de ligadura), L (el conjunto de vínculos interteóricos) y A (el conjunto de 'conjuntos borrosos admisibles', de suerte que el núcleo $\mathrm{K}$ del elemento teórico tiene la forma:

$$
\mathrm{K}=\left\langle\mathrm{M}_{\mathrm{p}}, \mathrm{M}, \mathrm{M}_{\mathrm{pp}}, \mathrm{C}, \mathrm{L}, \mathrm{A}\right\rangle
$$

\footnotetext{
${ }^{6}$ Pienso que es un error categorial identificar la clase de referencia de una teoría, es decir, aquello de que trata una teoría, con su ontología, pues, como bien lo señala M. Bunge, "una ontología no es un conjunto de cosas, sino una teoría filosófica relativa a las características básicas del mundo" Para él, "[e]strictamente hablando, una ontología de una teoría científica es el conjunto de hipótesis ontológicas (metafísicas o cosmológicas) presupuestas por la teoría” (Bunge 1976, p. 46).

7 Los estructuralistas proponen una variante para el concepto bourbakiano de estructuras conjuntistas a fin de proporcionar un marco general para hablar acerca de la clase de modelos para las teorías y elucidar la distinción entre los modelos potenciales y los modelos actuales (o simplemente modelos) de las teorías empíricas: "la clase de modelos potenciales de una teoría dada está determinada por especies de estructuras [estructuras de una especie determinada] cuyas formulas constitutivas $A_{j}$ todas expresan tipificaciones y nada más que tipificaciones. Por otro lado, la clase de modelos actuales estaría determinada por especies de estructuras determinadas no sólo por tipificaciones, sino también por "leyes reales" (Balzer, Moulines \& Sneed 1987, p. 13). Los modelos potenciales -como el primer sillar de una teoría empírica- poseen dos propiedades: a) poseen una estructura de una especie determinada; b) estas estructuras son de idéntico tipo para todos los $\mathbf{M}_{\mathrm{p}}$ de una teoría.
} 
Lo anterior significa que la identidad formal de cualquier teoría empírica con un cierto grado de complejidad incluye, dentro de la estructura del núcleo $\mathrm{K}$, los componentes anteriormente listados. Como lo muestra Moulines (Balzer \& Moulines 1996, pp. 7-8) sus relaciones (incluyendo las relaciones entre modelos actuales y los modelos potenciales), son como sigue:
a) $\mathbf{M} \subseteq \mathbf{M}_{\mathrm{p}}$
b) $\mathbf{M} \subset \operatorname{Pot}\left(\mathbf{M}_{\mathrm{p}}\right)$
c) Para cualquier $\lambda \in \mathrm{L}$ existe un $\mathbf{M}_{\mathbf{p}}^{\prime} \neq \mathbf{M}_{\mathrm{p}}$ tal que $\lambda \in \mathbf{M}_{\mathrm{p}} \times \mathbf{M}_{\mathrm{p}}^{\prime}$,
d) Existe una función muchos-uno $\mathbf{r}$ tal que $\mathbf{r}: \mathbf{M}_{\mathbf{p}} \rightarrow \mathbf{M}_{\mathbf{p p}}$,
e) $\mathbf{A} \subseteq U \in \operatorname{Pot}\left(\mathbf{M}_{\mathbf{p}} \times \mathbf{M}_{\mathbf{p}}\right)$.

Como se puede ver, todos los componentes del núcleo $\mathbf{K}$ (con excepción, claro está de los $\mathbf{M}_{\mathbf{p}}$ ) están caracterizados formalmente con relación a los modelos potenciales, $\mathbf{M}_{\mathbf{p}}$.

Un modelo potencial, $\mathbf{M}_{\mathbf{p}}$, del núcleo $\mathbf{K}$ es una estructura teórico-conjuntista (tuplo- $k+n$ ) del tipo

$$
x=\left\langle D_{1}, \ldots, D_{k}, R_{1}, \ldots, R_{n}\right\rangle
$$

donde los $D_{1}, \ldots, D_{k}$ son conjuntos (no-vacíos) que representan los "dominios de base" de la estructura (la mal llamada "ontología" de $\mathrm{T}$ ) y los $R_{1}, \ldots, R_{n}$ las relaciones (funciones, operaciones) construidas o tipificadas (en el sentido de la teoría de conjuntos) sobre los conjuntos de base. Los modelos potenciales, $\mathbf{M}_{\mathrm{p}}$, son aquellas estructuras (tuplo- $k+n$ ) que satisfacen los axiomas impropios (tipificaciones) de $\mathbf{T}$, pues los modelos (los modelos actuales), M, son los que satisfacen, además de las tipificaciones, las constricciones no meramente lógicas (leyes y ligaduras); los modelos potenciales parciales o, simplemente, modelos parciales, $\mathbf{M}_{\mathrm{p} p}$, son los modelos que se obtienen recortando (mediante la función recorte " $\mathbf{r}$ ") todas las funciones $\mathbf{T}$-teóricas en los $\mathbf{M}_{\mathrm{p}}$, i.e., aquellas funciones para cuya determinación o medición se presupone la aplicabilidad de $\mathbf{T}$; las condiciones de ligadura, $\mathrm{C}$, indican que los modelos de una y la misma teoría no se encuentran aislados, sino mutuamente relacionados por ciertas condiciones de segundo orden que se llaman 'constraints' (condiciones de ligadura); loa vinculos o relaciones interteóricos, $\mathbf{L}$, indican que las teorías mismas no son unidades aisladas, lo que significa que los modelos de las distintas teorías están conectados mutuamente y, finalmente, la aproximación, $\mathrm{A}$, indica que las teorías empíricas sólo son realmente aplicables dentro de cierto grado aceptable de aproximación, de suerte que "cualquier elucidación del concepto de teoría empírica debe dar cuenta del componente aproximativo; de lo contrario, la elucidación será necesariamente incompleta" (Moulines 1982, p. 166). ${ }^{8}$

No obstante, la reconstrucción modelo-teórica estructuralista difiere de otras reconstrucciones modelo-teóricas (en especial, de la de P. Suppes y colaboradores en la Universidad de Stanford), al establecer que las teorías empíricas son diferentes e incluso más complejas que las teorías puramente matemáticas, de suerte que en ellas se hace necesario introducir, como un componente esencial de su definición, las aplicaciones pretendidas e intencionales, I, de las que habló Adams, cuya identidad no se puede establecer formalmente, sino sólo histórico-pragmáticamente. Así, en un primer análisis como ya se advirtió-, un elemento teórico $\mathrm{T}$ es, desde el punto de vista estructuralista, un par ordenado $\langle\mathbf{K}, \mathbf{I}\rangle$, donde $\mathbf{I}$ es el conjunto de aplicaciones intencionales o pretendidas del elemento teórico T, i.e., el conjunto de aplicaciones a las que los usuarios del elemento teórico (los individuos o comunidades científicas) "tienen la intención" de aplicar el núcleo K. Las aplicaciones intencionales I se conciben como una subclase de una especie de estructuras conjuntistas $\mathbf{M}_{\mathrm{pp}}$ del elemento teórico $\mathbf{T}$

\footnotetext{
8 Es conveniente señalar que dentro de la clasificación de los diversos tipos de aproximación que propone Moulines, existen -además de la aplicación aproximativa de las teorías empíricas- otros tipos de aproximación como son las de "idealización" o "simplificación" en la construcción de los modelos, las relaciones puramente teóricas entre leyes como el caso de la ley de Galileo como aproximación de la ley de la gravitación y la aproximación interteórica entre dos teorías generales, i.e., entre estructuras globales con marcos conceptuales diferentes y con leyes fundamentales distintas, como el caso - reconstruido formalmente por Moulines- de la reducción de las leyes de Kepler a la teoría gravitacional de Newton (Moulines 1982, pp. 204-224).
} 
y, en consecuencia, son estructuras no-teóricas de T. Lo único que formalmente podemos decir de ellas es que son una subclase de los $\mathbf{M}_{\mathrm{pp}}$ : $\mathbf{I} \subseteq \mathbf{M}_{\mathrm{pp}}$. ${ }^{9}$

El par ordenado $\langle\mathbf{K}, \mathbf{I}\rangle$ que identifica cada elemento teórico está asociado con una aserción empírica ("empirical claim") que expresa la "pretensión empírica" de que ciertas aplicaciones intencionales I descritas T-no teóricamente, i.e., mediante términos/conceptos de teorías previamente disponibles, individualmente satisfacen las leyes y, colectivamente, las condiciones de ligadura ("constraints"), i.e., son modelos, M. La aserción empírica hace que las teorías (en forma derivativa o laxa) puedan ser, falsables (en el sentido popperiano de la 'falsabilidad') lo que hace que el estructuralismo -como lo anota Sneed- no sea "una nueva versión del instrumentalismo" (Sneed 1983, p. 351). ${ }^{10}$

\section{3.}

Van Fraassen y los estructuralistas tienen, como vimos, una manera diferente de concebir (matemáticamente) los modelos y aunque en el primero el grado de formalización de los diferentes componentes de la teoría es casi inexistente, sin embargo, en ambas propuestas modelísticas existe un marcado interés por los problemas de la aplicación de las teorías empíricas, i.e., por las relaciones entre el núcleo formal de teorías empíricas y los representantes de los sistemas físicos que, como 'entidades exteriores' no son, por su parte teorías; ambos distinguen, en cada teoría empírica, la parte 'teóricamente pura' de la que corresponde a las aserciones o hipótesis empíricas cuya forma lógica, en ambos casos, es la de un enunciado existencial.

Van Fraassen nos habla de la existencia de un conjunto de fenómenos observables (fenómenos de laboratorio) de suerte que la teoría es empiricamente adecuada y, por ende, la aserción o hipótesis empírica que la acompaña verdadera, si dichos fenómenos observables son isomorfos a la subestructura de uno de los modelos de la teoría. Ex profeso no hablo en términos contrafácticos porque van Fraassen dice que se está refiriendo a fenómenos observables realmente existentes y no a fenómenos observables que podrian existir.

Sneed, por su parte, habla de la existencia de estructuras no-teóricas, i.e., de $\mathbf{M}_{\mathrm{pp}}$ de una teoría, $\mathbf{T}$, que son extendibles a un modelo parcial de $\mathrm{T}$ y que, extendidas, son modelos (actuales) de $\mathrm{T}$, i.e., satisfacen las leyes y las condiciones de ligadura ("constraints") de $\mathrm{T}$, vale decir, las restricciones de $\mathrm{T}$.

Sin embargo, la principal diferencia de la aserción empírica conectada a la teoría entre el empirismo constructivo de van Fraassen y el estructuralismo sneediano es que mientras, en el primero, la distinción observable-inobservable (de las entidades, no de los términos) es epistemológica, en el segundo la distinción T-teórico/T-no-teórico (de los términos, no de las entidades) es funcional y, por tanto, hasta cierto punto pragmática, en la medida en que se basa en el funcionamiento de los términos/conceptos en cada teoría. El estructuralismo como dice W. Diederich, "es básicamente neutral en este aspecto” (se refiere al aspecto epistemológicas de las teorías), a pesar de que a la aserción que acompaña a la teoría la llama "aserción empírica" ("empirical claim") (Balzer \& Moulines 1996, p. 18).

La epistemología del empirismo constructivo de van Fraassen, como ya fue dicho, depende de una noción epistemológica de 'observabilidad', en la medida en que, para van Fraassen, "son las subestructuras observables de los modelos las que nos permiten decidir si una teoría propuesta es empíricamente adecuada o no" (Moulines 2006, p. 127). Tal noción de 'observabilidad' van Fraassen no duda en calificarla de "antropomórfica", aunque, hay que decirlo, es más o menos consciente de las

\footnotetext{
${ }^{9}$ W. Balzer, siguiendo un camino diferente al de J. D. Sneed considera que los sistemas reales los representamos mediante dos componentes: a) el conjunto de sistemas intencionales, I, que sólo contiene nombres (como símbolos interpretados) para los diferentes sistemas reales a los que se aplica la teoría, y b) el conjunto de datos, $\mathbf{D}$, recopilados mediante observación, medición y experimentos y que deben formularse en el lenguaje de la teoría. Balzer considera que "mediante una adecuada recopilación en listas (conjuntos) todo el conjunto de datos se puede transformar en una subestructura, i.e., en una subestructura de un modelo potencial de la teoría. Al resultado de esta recopilación de datos la llamamos una estructura de datos" (Balzer 1997, p. 289).

${ }^{10} \mathrm{La}$ falsabilidad a la que se alude es la de la aserción empírica y no la de las leyes y, en particular, de las leyes fundamentales, pues éstas, como se sabe, son empíricamente irrestrictas, salvo si están acompañadas de otras leyes especiales.
} 
dificultades que ello implica, en particular, cuando se trata de entidades que no son detectables por el Homo Sapiens, pero sí mediante instrumentos.

Las subestructuras de los modelos que constituyen la base empírica de contrastación de las teorías están, como ya se dijo, 'sub-determinadas' ('infra-determinadas') por los fenómenos. Ellas contienen nociones "puramente observacionales", en el sentido de van Fraassen, donde "lo que es observable es una cuestión independiente de la teoría", pues sólo "está en función de los hechos acerca de nosotros qua organismos, y estos hechos pueden incluir hechos acerca de los estados psicológicos que están presentes en la contemplación de la teoría" (van Fraassen 1996, p. 82). No obstante, lo que nos parece problemático es que las subestructuras, destinadas a constituir la base empírica de contrastación de las teorías científicas, estén constituidas por nociones "puramente observaciones", o como dice van Fraassen, por "observacionales básicos, como serían, en el caso de la mecánica clásica de partículas, por ejemplo, las mediciones de cantidades observables que son funciones de tiempo y espacio: velocidades y aceleraciones, pues las subestructuras empíricas o los 'modelos de datos' (expresión que toma de Suppes y a que hace referencia en Laws and Symmetry 1989) son más complejos de lo que nos podemos imaginar cuando nos referimos a lo "directamente observable". Las subestructuras a que hacemos referencia en la mecánica clásica de partículas son subestructuras cinemáticas, constituidas, entre otras cosas, por las nociones de 'tiempo continuo' y de 'distancia' como magnitud dos veces diferenciable (en el sentido del cálculo diferencial) sobre el tiempo y ninguno de los requisitos de 'continuidad' y de 'diferenciabilidad' corresponden al sentido de 'observabilidad' antropomórfico mencionado por van Fraassen (Moulines 2006, p. 128).

En consecuencia, si la base epistemológica del empirismo constructivo es la noción de 'observabilidad' y esta resulta problemática para la sub-determinación empírica de las subestructuras que son la base empírica contrastacional de las teorías, entonces las condiciones que propone van Fraassen para establecer la adecuación empírica de las teorías, a saber, que exista un modelo de la teoría tal que todos los fenómenos observables sean isomorfos a subestructuras de ese modelo, también resultaría problemático. Por lo tanto, se hace necesario apelar a otra forma de caracterización de los modelos contrastacionales de las teorías empíricas que, sin desconocer el carácter constructivo de la actividad científica (los modelos se construyen, no se descubren), ${ }^{11}$ supere las dificultades de una caracterización epistemológica como la del punto de vista empirista defendido por el 'empirismo contractivo' de van Fraassen que, aunque difiere del empirismo clásico y del empirismo de los positivistas lógicos del Círculo de Viena, no escapa a muchas de las objeciones a estos planteadas.

\section{4.}

El estructuralismo propone una solución al problema de la contrastación empírica de las teorías que supera las dificultades, tanto de la filosofía lógico-empirista de la ciencia, como de los defensores del llamado "empirismo constructivo". La solución propuesta encierra el famoso "problema de los términos teóricos' tan pronto como dichos términos se encuentran involucrados en las descripciones de los sistemas a los que las teorías se pretenden aplicar. Sneed en The Logical Structure of Mathematical Physics (1971) propone una solución a dicho problema mediante la reconstrucción de la aserción empírica de la teoría (elemento teórico) T como una afirmación global respecto de un tipo de sistemas (los modelos parciales) que podemos describir sin necesidad de apelar a los conceptos T-teóricos, es decir, a aquellos conceptos para cuya determinación (medición para el caso de funciones métricas) ${ }^{12}$ no es necesario presuponer por la validez de la teoría en cuestión, superando así las dificultades de la autojustificación y del regreso infinito.

\footnotetext{
${ }^{11}$ Van Fraassen alude a dos niveles constructivos: a) el de los sistemas físicos que, en sentido estricto, son idealizaciones de los fenómenos empíricos, y b) el de los 'modelos de datos' que resultan de mediciones, de outputs tecnológicos, etc.

${ }^{12}$ Los estructuralistas hablan de "determinación" de un concepto y no sólo de "medición”, pues la segunda sólo se aplica a funciones métricas y no a conceptos cualitativos: "La determinación incluye la medición pero no viceversa” (Balzer, Moulines \& Sneed 1987, p. 56).
} 
Tal distinción difiere, como es fácil advertirlo, de la distinción semántica absoluta 'teóricoobservacional' de la filosofía clásica de la ciencia y de la distinción epistemológica 'observable/no observable' de van Fraassen. La distinción que propone Sneed es, ante todo, pragmática y relativa a cada teoría (local), pues un término/concepto puede ser T-teórico en una teoría (elemento teórico) y Tno-teórico en otra. Ésta no sólo difiere de la distinción semántica absoluta (teórico/observacional) de la filosofía estándar de la ciencia y de la distinción epistemológica (observable/no observable) de van Fraassen, sino que, además, resulta de particular importancia para el análisis estructuralista de la contrastación de teorías, pues la aserción empírica que acompaña a la teoría nos dice que un conjunto de sistemas empíricos descritos T-no-teóricamente se comportan en la forma que establecen las constricciones que establece la teoría (leyes y condiciones de ligadura) cuando a ellos se le adicionan parámetros T-teóricos, i.e., son modelos de la teoría, en tanto son subsumibles por o encajan en la teoría, T. En otras palabras, si entre los modelos $\mathbf{M}_{\mathrm{pp}}(\mathrm{T})$ están todas estructuras de datos posibles, lo que la aserción empírica dice es que existe un conjunto de estructuras de datos posibles que, adicionadas o enriquecidas mediante funciones T-teóricas, son $\mathbf{M}(\mathrm{T})$. Formalmente la aserción empírica (enunciado Ramsey-Sneed) que corresponde a cada elemento teórico tendría la siguiente forma lógica:

$$
\exists \chi(X E A \wedge \chi \subseteq \mathrm{M} \wedge \chi \in \mathrm{C}),
$$

donde ' $\chi$ ' representa el conjunto modelos potenciales, $\mathbf{M}_{\mathrm{p}}$, que resultan de la operación de "extensión" $E$, i.e., de la adición de funciones T-teóricas, sobre los $\mathbf{A}\left(\mathbf{A} \subseteq \mathbf{M}_{\mathrm{pp}}\right)$ que representa el conjunto de aplicaciones intencionales del elemento teórico T, i.e., el conjunto de subestructuras (estructuras de datos posibles qua estructuras no-teóricas) cuyos elementos (idealizados) se pretenden explicar mediante el elemento teórico T. Lo que dice esta formulación es que existen conjuntos de modelos potenciales $X$ que son extensiones de modelos parciales, que satisfacen las leyes y las condiciones globales de ligadura, i.e., son modelos del elemento teórico. Una idea similar idea (aunque no idéntica) J. Zamora (2005, p. 212) la formula así:

$$
\exists x \in \mathrm{A} \exists y \in \operatorname{Ex}(x)(\mathrm{T} y \wedge \forall z \in \mathrm{A} \exists ! w \in \operatorname{Ex}(z)(\mathrm{T} x \wedge \mathrm{Cyx})) .
$$

Lo que Zamora afirma es que existe una aplicación empírica de la teoría $\mathrm{T}$ y existe una forma de extenderla (complementarla) mediante valores de ciertas funciones T-teóricas adicionales, tal que se cumplen dos cosas: a) que el sistema resultante de esta extensión es un modelo de $\mathbf{T}$ y, b) que para cualquier otra aplicación empírica existe una única extensión similar (recuérdese que " $\exists$ ! $x F x$ ” significa que "hay un solo $x$ tal que $F$ " que es una abreviatura de " $\exists x(F x \wedge y(F x \rightarrow x=y))$, de forma que los sistemas resultantes cumplen las leyes y las condiciones de ligadura $\mathbf{C}$ de $\mathbf{T}$, lo que significa que cada aplicación intencional de $\mathrm{T}$ puede ser convertida en un modelo $\mathbf{M}(\mathrm{T})$ que, además, es compatible con todos y cada uno de los modelos que resulten de la extensión de las demás aplicaciones.

Sin embargo, en esta formulación de Zamora no se hace referencia explícita al especto plurimodélico del 'enunciado Ramsey-Sneed', i.e., al 'enunciado Ramsey enmendado', sino a la versión monomodélica del enunciado Ramsey, cuando la reconstrucción del enunciado por parte de Sneed habla de la existencia de clases de aplicaciones, de suerte que el cuantificador existencial es un cuantificador de segundo orden; se cuantifica sobre variables de clases y no sobre variables de individuos.

No obstante, la aserción empírica tal como fue originalmente formulada por Sneed es insatisfactoria, porque estaría estableciendo una coincidencia exacta entre los valores medidos y los calculados y, como se sabe, en esta relación existen errores de aproximación. Se trata, entonces, de una versión idealizada (exacta) de la aserción empírica cuya modificación fue realizada y sistematizada por el estructuralismo a comienzos de la década de los 80 mediante la incorporación en ella del concepto de "aplicación aproximativa" a que hemos hecho referencia atrás.

Aunque esta reconstrucción modelo-teórica de la aserción empírica del estructuralismo y, la de Sneed, en particular, aparentemente guarda cierta similitud con la que propone van Fraassen, sin embargo, se hace necesario precisar dos aspectos que las diferencian: 
a) Pese a que van Fraassen y los estructuralistas comparten la idea de que los fenómenos empíricos (los 'sistemas reales') son ontológicamente distintos de su representación teórica, ya que ésta es una representación abstracta e idealizada de ellos, la manera como en cada caso se construyen dichas representaciones son diferentes. Para el empirismo constructivo van fraasseano, la construcción de las subestructuras empiricas, se lleva a cabo apelando a nociones (epistemológicas) "puramente observacionales" en el sentido de van Fraassen; para el estructuralismo, por el contrario, la construcción se hace apelando a términos/conceptos T-no-teóricos que, como vimos, nada tienen que ver, ni intensional ni extensionalmente, con las controversiales distinciones 'observable/no observable' de van Fraassen, o 'teórico/observacional' de lo que H. Putnam (1962) denominó "concepción heredada" ("received view") y para las que las clásicas críticas de H. Putnam (1962, pp. 240-251) y de P. Achinstein (1971) aún conservan su plena vigencia.

b) Dado que para van Fraassen y, hasta cierto punto, para los estructuralistas las teorías son identificables a través de una clase de modelos y no con una clase de modelos, se hace necesario formular (para su presentación/identificación), un enunciado o "aserción empírica" que dé cuenta de las "pretensiones empíricas" de los usuarios de las teorías (problema de la contrastación de las teorías), sin las dificultades de la "justificación circular" que se presentan en E. W. Adams.

En el caso de van Fraassen, la aserción empírica tiene que ver con la adecuación empirica de la teoría en la medida en que ésta se satisface syss existe un modelo de la teoría (modelo teórico) tal que todos los fenómenos observables sean isomorfos a subestructuras de este modelo, i.e., pueden ser subsumidas bajo un modelo. Como lo anota Moulines a propósito de lo que el llama el "representacionalismo" (2006, pp. 117-122), en vez del término matemático "isomorfismo", debería hablarse mejor de "homomorfismo" como una forma de relación más "débil" que el isomorfismo; el homomorfismo expresa una suerte de asimetría entre lo representado y la representación. Creo que los estructuralistas, en líneas generales, adoptarían este punto de vista, y hablarían de la "subsunción" como la "incrustación" ("embedding”) aproximada de un modelo empírico (la estructura no-teórica de T) en un modelo matemático (la estructura teórica de T), de suerte que, el primero, se concibe como una "subestructura" o "submodelo" ("sous modèle" como lo llama Moulines) del segundo, sólo que caracterizada no en términos de la distinción epistemológica "observable/inobservable", sino de la distinción funcional "T-teórico/T-no-teórico".

A pesar de algunas de las críticas formuladas a la solución sneediana del "problema de los términos teóricos", considero que la solución ofrecida por este autor a dicho problema es una solución altamente satisfactoria, pues no sólo permite dar cuenta del modo como las aplicaciones intencionales I satisfacen todos los requerimientos de la teoría sin caer en una circularidad viciosa o en un regreso infinito, sino porque, con ella, se superan las dificultades de la distinción "observable/no observable" de van Fraassen y, en especial, su afirmación de que "lo observable es una cuestión independiente de la teoría" (van Fraassen 1996, p. 32) sobre todo cuando él mismo, con anterioridad a esta afirmación, en el mismo texto donde hace tal afirmación (que más parece de un realista que de un antirrealista), ha planteado, con claridad meridiana, la necesidad de no confundir el observar con el observar que y que, para precisar, ilustra con la situación (imaginaria) de un hombre de la Edad de Piedra recientemente encontrado en Filipinas a quien se le entrega una pelota de tenis y la tira, sin ver que (observar que) es una pelota de tenis, pues carece de esos conceptos. Decir que alguien observó una pelota de tenis no implica, en lo más mínimo, que alguien observó que era una pelota de tenis, pues, para ello, se requeriría algún conocimiento conceptual de lo que es un juego de tenis.

Finalmente y, para concluir, debo señalar que esta distinción del observar y del observar que nos coloca frente al problema de la discusión realismo/antirrealismo en las teorías empíricas. Sin negar la existencia de objetos/fenómenos empíricos como algo que existe en y con independencia del lenguaje, o, de modo más general, de las representaciones (percepciones, pensamientos, creencias, deseos, etc.) y que corresponde a lo que en otro escrito denominé "realismo ontológico" o "externo" (como lo 
denomina J. Searle), tenemos que afirmar que, en el caso de las teorías, la realidad de los sistemas objeto de descripción de la teoría, no es algo dado como lo proponen los realistas y, de modo especial, los realistas científicos para quienes el mundo (el universo) no sólo existe con independencia de nosotros (y en esto coincidiría con el realismo ontológico o externo), sino que, además, posee una organización, i.e., está estructurado de una y sólo una manera, de suerte que la función de las teorías científicas consiste en descubrir ese orden o estructura ya dado en el mundo. A esta segunda forma de realismo ontológico la he llamado realismo ontológico (externo) fuerte, para diferenciarla del realismo ontológico (externo) débil que sólo se compromete con la existencia de los objetos/fenómenos/acontecimientos, pero no con la creencia de que, con independencia de los marcos conceptuales que llamamos "teorías", dichos objetos tengan una organización o estructura. Otro asunto bien diferente es el del realismo epistemológico cuyas dos tesis básicas serían: a) las teorías proporcionan un conocimiento objetivo del mundo, y b) ese conocimiento objetivo es verdadero o aproximadamente verdadero. El error del realismo ontológico fuerte consiste en confundir los planos ontológico y epistemológico, pues que el mundo posea una determinada estructura no es un problema ontológico, sino epistemológico, o mejor, epistemosemántico, pues la organización del mundo no es una cuestión ontológica , sino algo que depende del modo como describamos/interpretemos la realidad. Lo que van Fraassen denomina el "alcance pretendido de la teoría" y los estructuralistas "aplicaciones intencionales I", no son sistemas físicos, sino representaciones altamente idealizadas y contrafácticas de esos sistemas físicos y es respecto de esos sistemas idealizados y contrafácticos que la teoría satisface sus demandas de explicación y de predicción mediante la construcción de modelos (teóricos) a los que las descripciones de los fenómenos (subestructuras empíricas) se deben ajustar. El acotamiento del campo de objetos de las teorías empíricas (los sistemas físicos) sólo es posibles si existen marcos conceptuales y ellos sólo permiten representar aquellos componentes que son captados por los conceptos fundamentales de la teoría. Razón tiene Moulines cuando afirma que las teorías son interpretaciones y propone, como definición intuitiva y provisional de "interpretación de cierto dominio de objetos": la "incrustación" en ese dominio, de modo consciente y deliberado, de un aparato conceptual elaborado previamente que nos permite "reconstruir" ese dominio (o mejor, ciertos aspectos de éste; Moulines 1982, pp. 44-45). El objetivo de la ciencia es construir modelos (modelos teóricos) a los que se ajusten las descripciones Tno-teóricas de los fenómenos qua construcciones abstractas e idealizadas y no modelos a los que se ajusten los fenómenos qua fenómenos observables, máxime si se tiene en cuenta el polémico sentido que de "observable" propone van Fraassen.

Pero en este proceso de "reconstrucción" de cierto dominio de objetos Anjan Chakravartty (2001, pp. 325-345) ve conveniente distinguir "abstracción" e "idealización”. La primera es el proceso a través del cual sólo algunos de los muchos factores o parámetros relevantes presentes en la realidad son incorporados al modelo que corresponde a una clase particular de fenómenos. Según Chakravartty esto se hace por dos razones: a) porque el número de parámetros potencialmente (relevantes) para una clase dada de fenómenos empíricos es tan extremadamente grande, que construir un modelo con tan elevando número de parámetros resultaría impráctico, y b) porque la influencia de muchos de esos parámetros potencialmente (relevantes) es generalmente despreciable respecto de la clase de fenómenos definidos por nuestros intereses en una particular investigación teórica o práctica. Pero si la abstracción tiene que ver con la escogencia de algunos parámetros y la ignorancia de otros, la idealización tiene que ver con la simplificación de los parámetros particulares escogidos. Asumir en la mecánica clásica la concentración de las masas de los cuerpos en puntos inextensos es una idealización. En estos casos, estaríamos hablando de situaciones contrafácticas como si la masa de los cuerpos (el Sol, por ejemplo) estuviera concentrada en un punto inextenso: el punto de masa del Sol.

De la distinción entre "abstracción" e "idealización”, Chakravartty extrae como consecuencia que algunos aspectos un mismo modelo puede ser visto realísticamente, mientras que otros sólo instrumentalmente (anti-realísticamente). Así, un modelo que emplea la abstracción puede ser considerado realísticamente, pues los parámetros seleccionados tienen su contraparte en el mundo, así existan otros parámetros que no fueron seleccionados en el modelo. Pero esta consideración realista no 
puede ser adoptada en el caso de la idealización ya que "los modelos contradicen lo que consideramos verdadero de la realidad" (Chakravartty 2001, p. 329), aunque, claro está, en este caso es necesario diferenciar la idealización de la ficción, pues "nosotros estamos autorizados al menos a creer en la existencia de esos aspectos de la realidad que han sido idealizados" (Chakravartty 2001, p. 329). Aquí, por supuesto estaríamos hablando de lo que I. Hacking llama "realismo de las entidades" como algo diferente del "realismo de las teorías" donde lo que interesa es establecer si las teorías "son verdaderas, o si son verdaderas-o-falsas, o si aspiran a la verdad" (Hacking 1996, p. 45).

Reconocer que las teorías se ocupan directamente de fenómenos abstractos, idealizados y contrafácticos y no de fenómenos empíricos, plantea un problema acerca del papel que, por ejemplo, desempeñan las operaciones de laboratorio en la contrastación de las teorías, toda vez que la base empírica teorizada, la de los modelos parciales, es paradójicamente, empíricamente inexistente, justamente porque los sistemas que son su referente (correlato) son abstracciones e idealizaciones. Quizás convenga hablar -como lo propone Rodríguez Ladreda (1993)- de una base empírica precientífica, descrita operacionalmente y que, a la manera del 'lenguaje observacional neutro' de los empiristas-lógicos, no dependa de ninguna teoría en la que los modelos parciales son modelos, ni de ninguna otra. Reconocer este tipo de 'escenario observacional' de tipo operacional, pese a su problematicidad, pueda contribuir a resolver el tradicional problema de la verdad como adecuación y a salir de lo que aparece como el cul de sac del realismo interno. Igualmente, permitiría resolver problemas como los de la comparación de teorías en los casos de pares de teorías inconmensurables. No obstante, creo que sigue siendo un problema abierto y por ahora nos basta con reconocer que, si se adopta una visión 'modelo-teórica' de las teorías, resulta imposible predicar primariamente la verdad o la falsedad de una teoría, pero si en forma derivativa cuando su aserción empírica correspondiente resulta verdadera, i.e., cuando las subestructuras de los modelos potenciales enriquecidas con términos/conceptos T-teóricos satisfacen aproximadamente las leyes y demás restricciones de la teoría, i.e., encajan en la teoría.

Para concluir, pienso que, aunque existen diferencias entre el empirismo constructivo y el estructuralismo en muchos aspectos, sin embargo, de su intercambio, el primero debería aprender que cierto grado de formalización le ayudaría a precisar mejor algunos aspectos básicos de su concepción, y, el segundo, realizar consideraciones epistemológicas conectadas con el uso de los modelos, como en su momento lo propuso W. Diederich. Quizás valga la pena recordar estas palabras de Whitehead (1967, p. 186): "A clash of docrines it not a disaster, it is an oportunity" ("Un choque de doctrinas no es un desastre, es una oportunidad"). 
Achinstein, P. (1971), Concepts of Science, Baltimore: John Hopkins Press.

Balzer, W. ([1982] 1997), Teorias empiricas: modelos, estructuras y ejemplos, Madrid: Alianza.

Balzer, W. y C. U. Moulines (eds.) (1996), Structuralist Theory of Science. Focal Issues, New Results, Berlin: de Gruyter.

Balzer, W., Moulines, C. U. y J. D. Sneed (1987), An Architectonic for Science - The Structuralist Program, Dordrecht: Reidel. (Versión castellana de Pablo Lorenzano: Una arquitectónica para la ciencia. El programa estructuralista, Bernal: Universidad Nacional de Quilmes, 2012.)

Bunge, M. (1976), Tratado de Filosofía Básica 1. Semántica, São Paulo: EPU/EDUSP.

Chakravartty, A. (2001), “The Semantic or Model-Theoretic View of Theories and Scientific Realism”, Synthese 127: 325-345.

Da Costa, N. (2000), El conocimiento científico, México: U.N.A.M.

Dalla Chiara, M. y G. Toraldo di Francia ([1999] 2001), Confines: Introducción a la filosofía de la ciencia, Barcelona: Crítica.

Díez, J. A. y C. U. Moulines (1997), Fundamentos de Filosofía de la Ciencia, Barcelona: Ariel.

Hacking, I. ([1983] 1996), Representar e intervenir, México/Barcelona: UNAM/Paidós.

Nagel, E., Suppes, P. y A. Tarski (eds.) (1962), Logic, Methodology and Philosophy of Science-Proceedings of the 1964 International Congress for Logic, Methodology and the Philosophy of Science, Amsterdam: North Holland.

Lawvere, F. W. y S. Schanuel ([1997] 2002), Matemáticas conceptuales, México: Siglo XXI.

Moulines, C. U. (1982), Exploraciones metacientificas, Madrid: Alianza.

Moulines, C. U. (1991), Pluralidad y recursión. Estudios epistemológicos, Madrid: Alianza.

Moulines, C. U. (ed.) (1993), La ciencia: estructura y desarrollo, Madrid: Trotta.

Moulines, C. U. (2006), La philosophie des sciences. L'invention d'une discipline (fin XIXe-début XXIe siècle), Paris: Éditions Rue d'Ulm/Presses de l'École normale supérieur.

Perdomo, I. y J. Sánchez (2003), Hacia un nuevo empirismo, Madrid: Biblioteca Nueva.

Pérez Ransanz, A. R. (1985), “El concepto de teoría empírica según van Fraassen”, Crítica 17(51): 3-19.

Putnam, H. (1962), "What Theories Are Not", en Nagel, Suppes \& Tarski (1962), pp. 240-251.

Rodríguez Ladreda, R. M. (1993), Teoría y práctica en la ciencia, Granada: Universidad de Granada.

Sneed, J. D. (1971), The Logical Structure of Mathematical Physics, Dordrecht: Reidel.

Sneed, J. D. (1983), "Structuralism and Scientific Realism", Erkenntnis 19: 345-370.

van Fraassen, B. (1979), “On the Extensión of Beth's Semantics of Physical Theories”, Philosophy of Science 37: 325-339.

van Fraassen, B. (1985), “On the Question of Identification of a Scientific Theory (A Replay to 'Van Fraassen's Concept of Empirical Theory' by Pérez Ransanz)", Crítica 17(51): 21-29.

van Fraassen, B. (1996 [1980]), La imagen cientifica, México: Paidós/UNAM.

Whitehead, N. W. (1967), Science and the Modern World, New York: The Free Press.

Zamora, J. (2005). Cuestión de protocolo. Ensayos de metodología de la ciencia, Madrid: Tecnos. 